\title{
On subset sums of a fixed set
}

\author{
by \\ Yong-GaO Chen (Nanjing)
}

1. Introduction. Let $A$ be a strictly increasing sequence of positive integers. Let $P(A)=\left\{\sum \varepsilon_{i} a_{i}: a_{i} \in A, \varepsilon_{i}=0\right.$ or 1$\}$ be the set of all the subset sums of $A$. $A$ is said to be subcomplete if $P(A)$ contains an infinite arithmetic progression. P. Erdős conjectured that if $a_{n+1} / a_{n} \rightarrow 1$, then $A$ is subcomplete. But J. W. S. Cassels [1] proved that for every $\varepsilon>0$ there exists a sequence $A$ for which $a_{n+1}-a_{n}=o\left(a_{n}^{1 / 2+\varepsilon}\right)$ and $A$ is not subcomplete. Let $A(n)=A \cap[1, n]$. The cardinality of a finite set $S$ is denoted by $|S|$. In 1962 Erdős [2] proved that if $|A(n)|>c n^{(\sqrt{5}-1) / 2}(c>0)$, then $A$ is subcomplete. In $1966 \mathrm{~J}$. Folkman [3] improved this to $|A(n)|>n^{1 / 2+\varepsilon}(\varepsilon>0)$, and recently, N. Hegyvári [5] showed $|A(n)|>300 \sqrt{n \log n}$ for $n>n_{0}$. A similar result was also proved by Łuczak and Schoen [6] independently. In this note we improve Hegyvári's result:

ThEOREM 1. There exists an absolute constant $C>1$ such that if $A=$ $\left\{a_{1}<a_{2}<\ldots\right\}$ is an infinite sequence of positive integers with $|A(n)|>$ $C \sqrt{n}$ for $n>C^{2}$, then $A$ is subcomplete.

COROLlary. There exists an absolute constant $c>0$ such that if $A=$ $\left\{a_{1}<a_{2}<\ldots\right\}$ is an infinite sequence of positive integers with $a_{n}<c n^{2}$ for $n>c^{-1}$, then $A$ is subcomplete.

This is best possible (cf. [5]). The Corollary gives a partial answer to a question in [3].

\section{Preliminaries}

Lemma 1. Let $B=\left\{b_{1}<b_{2}<\ldots\right\}$ be an infinite sequence of positive integers, and let $n_{0}^{\prime}$,d be positive integers with $n_{0}^{\prime}>4$. Assume that $b_{i} \geq$

2000 Mathematics Subject Classification: 11B75, 11A67.

Supported by the National Natural Science Foundation of China, Grant No. 10171046 and the "333 Project" Foundation of Jiangsu Province of China. 
$i+4 d$ for all $i$ and $|B(n)| \geq 2 \sqrt{d n}$ for $n \geq n_{0}^{\prime}$. Then there exists an infinite sequence $\left\{v_{1}<v_{2}<\ldots\right\}$ in $P(B)$ for which $d \mid v_{n}$ and $v_{n+1}-v_{n}<d n_{0}^{\prime}$.

Proof. By the Erdős-Ginzburg-Ziv theorem (cf. [7]), for each $i \geq 1$, there exists $d$ integers $b_{i_{1}}, \ldots, b_{i_{d}}$ in $\left\{b_{2(i-1) d+1}, \ldots, b_{2 i d}\right\}$ such that $u_{i}=$ $b_{i_{1}}+\ldots+b_{i_{d}}$ is divisible by $d$. Then $u_{1}<u_{2}<\ldots$ Let $U=\left\{u_{1}, u_{2}, \ldots\right\}$ and $P(U)=\left\{v_{1}<v_{2}<\ldots\right\}$. Then $d \mid v_{n}$ for all $n$. Now we show that $v_{n+1}-v_{n}<d n_{0}^{\prime}$.

Since $|B(n)| \geq 2 \sqrt{d n}$ for $n \geq n_{0}^{\prime}$, it follows that if $b_{n} \geq n_{0}^{\prime}$, then $b_{n} \leq$ $n^{2} /(4 d)$. Hence $b_{2 d}<n_{0}^{\prime}$. Let $n_{1}$ be the largest integer with $b_{2 n_{1} d}<n_{0}^{\prime}$. Then $n_{1} \geq 1$. For $n \geq 1$, let $j_{1}$ be the least integer such that $\sum_{1 \leq i \leq j_{1}} u_{i}>v_{n}$; $j_{2}$ be the least integer such that $\sum_{1 \leq i \leq j_{2}} u_{i}>v_{n}-u_{j_{1}}$; and so on. Thus we have defined $j_{1}>\ldots>j_{t}=1$ such that

$$
\sum_{1 \leq i \leq j_{k}} u_{i}>v_{n}-u_{j_{1}}-\ldots-u_{j_{k-1}}, \quad k=2,3, \ldots, t .
$$

Define $u_{j_{0}}=0$. Since $u_{j_{0}}<v_{n}<u_{j_{0}}+u_{j_{1}}+\ldots+u_{j_{t}}$, there exists an integer $l$ with $0 \leq l<t$ such that

$$
u_{j_{0}}+u_{j_{1}}+\ldots+u_{j_{l}} \leq v_{n}<u_{j_{0}}+u_{j_{1}}+\ldots+u_{j_{l+1}} .
$$

Suppose that $j_{l+1}>n_{1}$. Then $b_{2 j_{l+1} d} \geq n_{0}^{\prime}$ and

$$
\begin{aligned}
v_{n}-u_{j_{0}}-\ldots-u_{j_{l}} & <u_{j_{l+1}} \leq d b_{2 j_{l+1} d} \leq d \frac{\left(2 j_{l+1} d\right)^{2}}{4 d} \\
& \leq d \sum_{1 \leq i \leq j_{l+1}-1}(2(i-1) d+4 d+1) \\
& \leq d \sum_{1 \leq i \leq j_{l+1}-1} b_{2(i-1) d+1} \leq \sum_{1 \leq i \leq j_{l+1}-1} u_{i}
\end{aligned}
$$

contrary to the definition of $j_{l+1}$. Hence $j_{l+1} \leq n_{1}$. Thus, by (1) and $j_{l+1}$ $\leq n_{1}$

$$
v_{n+1}-v_{n} \leq u_{j_{l+1}} \leq d b_{2 j_{l+1} d}<d n_{0}^{\prime} .
$$

This completes the proof of Lemma 1 .

LEMMA 2. Let $0<a_{1}<\ldots<a_{k} \leq n$ be an increasing sequence of integers. Assume that $n>2500$ and $k>200 \sqrt{n \log n}$. Then there exist integers $d, y, z$ such that $1 \leq d \leq 50 \sqrt{n / \log n}, z-y>\frac{3}{7} n \log n$ and

$$
\{t d: y \leq t \leq z\} \subset P\left(\left\{a_{1}, \ldots, a_{k}\right\}\right)
$$

Proof. By Theorem 4 in [8] (see also [4]) there exist integers $d, y, z$ with

$$
1 \leq d \leq 10^{4} n k^{-1}, \quad z \geq 7^{-1} \cdot 10^{-4} k^{2}, \quad y \leq 7 \cdot 10^{4} z n k^{-2}
$$

and

$$
\{t d: y \leq t \leq z\} \subset P\left(\left\{a_{1}, \ldots, a_{k}\right\}\right)
$$


For these $d, y, z$ we have

$$
1 \leq d \leq 50 \sqrt{\frac{n}{\log n}}, \quad z-y \geq 7^{-1} \cdot 10^{-4} k^{2}\left(1-7 \cdot 10^{4} n k^{-2}\right) \geq \frac{3}{7} n \log n .
$$

This completes the proof of Lemma 2.

Lemma 3. Let $A\left(D_{i}, H_{i}\right)=\left\{a_{i}+t D_{i}: 0 \leq t \leq H_{i}\right\}(i=0,1, \ldots, l)$ be arithmetic progressions of integers. Assume that

$$
H_{i} \geq D_{0}+D_{i+1}, \quad i=0,1, \ldots
$$

Then there exists an arithmetic progression $A\left(d_{l}, h_{l}\right)=\left\{a+t d_{l}: 0 \leq t \leq\right.$ $\left.h_{l}\right\} \subset A\left(D_{0}, H_{0}\right)+\ldots+A\left(D_{l}, H_{l}\right)$ with $d_{l}=\left(D_{0}, \ldots, D_{l}\right)$ and $h_{l} \geq H_{l}-D_{0}$.

This follows from the proof of Lemma 4 in [5].

3. Proof of Theorem 1. In this section we prove the following theorem which implies Theorem 1.

TheOREM 2. Let $A=\left\{a_{1}, a_{2}, \ldots\right\}$ be an infinite sequence of positive integers, $n_{0}$ be an integer with $n_{0} \geq e^{60}$ and $w=50 \sqrt{n_{0} / \log n_{0}}$. Assume that $|A(n)| \geq 202 \sqrt{12 w n}$ for $n_{0} \leq n \leq e^{12 w n_{0}}$ and $|A(n)| \geq 808 \sqrt{w n}$ for $n>e^{12 w n_{0}}$. Then $A$ is subcomplete.

Proof. Let $n_{i}=n_{i-1}^{2}(i=1,2, \ldots)$. Let $l$ be the integer with $n_{l-1}<$ $e^{6 w} \leq n_{l}$. Then $n_{l}=n_{l-1}^{2}<e^{12 w}$. Let $n_{-1}=0$. Hence

$$
\begin{aligned}
\left|A\left(n_{i}\right) \backslash A\left(n_{i-1}\right)\right| & \geq 202 \sqrt{n_{i} \log n_{i}}-n_{i-1} \geq 202 \sqrt{n_{i} \log n_{i}}-\sqrt{n_{i}} \\
& >200 \sqrt{n_{i} \log n_{i}}+1, \quad i=0,1, \ldots, l .
\end{aligned}
$$

Take $B_{i}=A\left(n_{i}\right) \backslash A\left(n_{i-1}\right)$ for $i=0,1, \ldots, l-1$ and $B_{l} \subset A\left(n_{l}\right) \backslash A\left(n_{l-1}\right)$ with $\left|B_{l}\right|=\left[200 \sqrt{n_{l} \log n_{l}}\right]+1$. By Lemma 2, for each $i$, there exists an arithmetic progression

$$
A_{i}=\left\{a_{i}+D_{i} k: 0 \leq k \leq H_{i}\right\} \subset P\left(B_{i}\right)
$$

with $D_{i} \leq 50 \sqrt{n_{i} / \log n_{i}}$ and $H_{i} \geq \frac{3}{7} n_{i} \log n_{i}$. Since

$$
H_{i} \geq \frac{3}{7} n_{i} \log n_{i}>50 \sqrt{\frac{n_{i+1}}{\log n_{i+1}}}+w \geq D_{i+1}+D_{0},
$$

by Lemma 3 there exists an arithmetic progression

$$
A(d, h)=\{a+d k: 0 \leq k \leq h\} \subset A_{0}+\ldots+A_{l}
$$

with $d \mid D_{0}$ and $h \geq H_{l}-D_{0}$.

For $n_{l} \leq n \leq e^{\overline{12} w}$ we have

$$
\begin{aligned}
\left|A(n) \backslash\left(B_{l} \cup A\left(n_{l-1}\right)\right)\right| & \geq 202 \sqrt{n \log n}-200 \sqrt{n_{l} \log n_{l}}-1-n_{l-1} \\
& \geq 2 \sqrt{n \log n_{l}}-1-\sqrt{n_{l}} \geq 2 \sqrt{6} \sqrt{w n}-1-\sqrt{n_{l}} \\
& \geq 2 \sqrt{w n} \geq 2 \sqrt{D_{0} n} \geq 2 \sqrt{d n} .
\end{aligned}
$$


For $n>e^{12 w}$ we have

$$
\begin{aligned}
\left|A(n) \backslash\left(B_{l} \cup A\left(n_{l-1}\right)\right)\right| & \geq 808 \sqrt{w n}-200 \sqrt{n_{l} \log n_{l}}-1-\sqrt{n_{l}} \\
& \geq 808 \sqrt{w n}-200 \sqrt{12} \sqrt{w n}-1-\sqrt{n_{l}} \geq 2 \sqrt{w n} .
\end{aligned}
$$

Let $A \backslash\left(B_{l} \cup A\left(n_{l-1}\right)\right)=\left\{b_{1}<b_{2}<\ldots\right\}$. Then

$$
b_{1} \geq n_{l-1}=\sqrt{n_{l}} \geq e^{3 w} \geq 4 w+1 \geq 4 D+1 \geq 4 d+1 .
$$

Hence $b_{i} \geq b_{1}+i-1 \geq i+4 d$. By Lemma 1 there exists an infinite sequence

$$
\left\{v_{1}<v_{2}<\ldots\right\} \subseteq P\left(A \backslash\left(B_{l} \cup A\left(n_{l-1}\right)\right)\right)
$$

with $d \mid v_{i}$ and $v_{i+1}-v_{i}<n_{l} d$. Since $D_{0}\left(n_{l}+1\right)<2 w n_{l}<\frac{3}{7} n_{l} \log n_{l}$, we have

$$
n_{l} d \leq n_{l} D_{0}<\frac{3}{7} n_{l} \log n_{l}-D_{0} \leq H_{l}-D_{0} \leq h .
$$

Hence

$$
\begin{aligned}
A(d, h)+\left\{v_{1}, v_{2}, \ldots\right\} & =\left\{a+v_{1}+d k: k=0,1, \ldots\right\} \\
& \subseteq P\left(B_{l} \cup A\left(n_{l-1}\right)\right)+P\left(A \backslash\left(B_{l} \cup A\left(n_{l-1}\right)\right)\right) \\
& \subseteq P(A) .
\end{aligned}
$$

This completes the proof of Theorem 2 .

Proof of the Corollary. Let $C$ be as in Theorem 1 and $c=\frac{1}{4} C^{-2}$. If $c i^{2} \leq n$, then $a_{i} \leq n$. Hence $|A(n)| \geq[\sqrt{n / c}] \geq[2 C \sqrt{n}]>C \sqrt{n}$. Then the Corollary follows from Theorem 1 .

4. Remark. I believe (but have no proof) that for every $K>0$ there exists an $n_{0}$ and a sequence $A$ which is not subcomplete such that $|A(n)| \geq$ $K \sqrt{n}$ for $n \geq n_{0}$.

\section{References}

[1] J. W. S. Cassels, On the representation of integers as sums of distinct summands taken from a fixed set, Acta Sci. Math. (Szeged) 21 (1960), 111-124.

[2] P. Erdős, On the representation of large integers as sums of distinct summands taken from a fixed set, Acta Arith. 7 (1962), 345-354.

[3] J. Folkman, On the representation of integers as sums of distinct terms from a fixed sequence, Canad. J. Math. 18 (1966), 643-655.

[4] G. Freiman, New analytical results in subset-sum problem, Discrete Math. 114 (1993), 205-218.

[5] N. Hegyvári, On the representation of integers as sums of distinct terms from a fixed set, Acta Arith. 92 (2000), 99-104.

[6] T. Łuczak and T. Schoen, On the maximal density of sum-free sets, ibid. 95 (2000), 225-229. 
[7] M. B. Nathanson, Additive Number Theory, Grad. Texts in Math. 165, Springer, New York, 1996.

[8] A. Sárközy, Finite addition theorems II, J. Number Theory 48 (1994), 197-218.

Department of Mathematics

Nanjing Normal University

Nanjing 210097, China

E-mail: ygchen@njnu.edu.cn

Received on 28.12.2000

and in revised form on 4.3.2002 